\title{
Symptomless myocarditis and myalgia in viral and Mycoplasma pneumoniae infections
}

Sir:

We are surprised at Drs. Lewes, Rainford, and Lane (British Heart fournal, 1974, 36, 924) seizing upon such a nonspecific symptom as myalgia as being a clue to any complication, much less being indicative of myocarditis. In our experience, myalgia accompanies viral influenza as regularly as a runny nose accompanies the common cold. Evanescent and minor ST-T wave changes are, also, nonspecific findings in the electrocardiogram in a host of illnesses and under many circumstances of health and, therefore, cannot be used to establish the diagnosis of myocarditis (Marriott, 1960; Lamb, 1965; Surawicz, 1972). We agree with Dr. Lewes and his colleagues that it is critical not to attribute non-diagnostic electrocardiographic changes in viral disease to myocardial infarction, but we would add that neither should a diagnosis of myocarditis be made on the basis of unwarranted clinical and electrocardiographic evidence. A diagnosis of myocarditis, in America at least, could keep a patient from obtaining life assurance or cause his premiums to be rated up.

Drs. Lewes, Rainford, and Lane made serious errors in their electrocardiographic technique, descriptions, and display. Because they only displayed single complexes, one cannot tell whether or not the nonspecific ST-T changes they presented are rate related; we are particularly concerned about the possibility of tachycardia producing some of the electrocardiographic abnormalities shown. In their article, they described both CR and Wilson chest leads ( $\mathrm{V}$ leads), but the tracings displayed are only labelled as limb and V leads. They presented no evidence to substantiate their statement that in their study the CR leads proved superior to the $V$ leads. It seems a tenuous diagnostic assumption in Cases $I$ and 2 to attribute short-lived and minor ST-T changes to myocarditis in feverish, vomiting patients. In Case 3, a 14-year-old boy, the interval between tracings was 2 years, and the change in $T$ waves from inverted to upright in the right heart leads could well be the normal transition from juvenile to adult pattern. Lateral wall $T$ wave inversions reverting to normal in a patient with left lower lobe pneumonia (Case 4) and the transient diphasic $T$ waves during acute fever in a patient with a history of prior paroxysmal tachycardia (Case 6) hardly permit a categorical diagnosis of myocarditis. However, their most striking example of alleged electrocardiographic abnormalities occurred in Case 5 of Fig. 5 of the same article in which the $V$ leads of 13.2 .69 are the mirror-image of the $V$ leads taken before (3.12.68) and after (24.2.69) this 'bizarre' tracing. The authors reassured the reader that there was 'no question of the leads being transposed', but to us no other explanation could account for the pattern seen. We agree that it is impossible to mirror-image the standard $\mathrm{V}$ leads by switching any limb lead with any chest lead. However, if CR leads are recorded instead of the Wilson $V$ leads, then inadvertent placement of the chest lead to the right arm and the right arm lead to the chest will produce this error exactly.

Daniel Simon,
Cardiovascular Research Institute,
University of California, San Francisco.

Joseph A. Abbott, Electrocardiographic Laboratory, San Francisco General Hospital, and Department of Medicine, University of California, San Francisco, U.S.A.

\section{References}

Lamb, L. E. (1965). Electrocardiography and Vectorcardiography. Instrumentation, Fundamentals, and Clinical Applications, p. 423. W. B. Saunders, Philadelphia.

Marriott, H. J. L. (1960). Coronary mimicry: normal variants, and physiologic, pharmacologic, and pathologic influences that simulate coronary patterns in the electrocardiogram. Annals of Internal Medicine, 52, $41 \mathrm{I}$.

Surawicz, B. (1972). The pathogenesis and clinical significance of primary T-wave abnormalities. In Advances in Electrocardiography, pp. 377-421. Ed. by R. C. Schlant and J. W. Hurst. Grune and Stratton, New York.

This letter was shown to Dr. David Lewes who replies as follows: 
Sir:

A diagnosis of myocarditis is irrefutable only when the proven acute onset of left ventricular disability is accompanied by biopsy confirmation of myocarditis at the time of the proven viral infection. It is self-evident that a diagnosis of myocarditis is otherwise tentative. There are no specific and irrefutable electrocardiographic changes in myocarditis. ... Our main thesis is that myalgia occurring in the course of a proved viral infection is accompanied by transitory and occasionally more prolonged ST changes in the electrocardiogram. These changes, we maintain, stand for an obligatory inferential diagnosis of myocardial involvement - a diagnosis which is not usually serious (as we have stressed) or of a future ill omen - nor one which would warrant such drastic responses as the rating up of life insurance premiums which appears to be the case in the New World.

David Lewes,

Bedford General Hospital (South Wing), Kempston Road, Bedford MK42 9DJ. 